\title{
Isolated Abducens Nerve Palsy due to a Dural Arteriovenous Fistula with Drainage into the Inferior Petrosal Sinus
}

\author{
Kazuto Tsukita ${ }^{a, b}$ \\ Haruhi Sakamaki-Tsukita ${ }^{\mathrm{a}, \mathrm{b}}$ \\ Toshihiko Suenaga ${ }^{a}$ \\ aDepartment of Neurology, Tenri Hospital, \\ Tenri, Nara, Japan \\ 'Department of Neurology, \\ Graduate School of Medicine, \\ Kyoto University, Kyoto, Japan
}

Dear Editor,

Abducens nerve palsy is the most common type of ocular nerve palsy to occur in isolation, and it has several associated etiologies. ${ }^{1}$ It is important to identify the causative lesion to determine the etiology of abducens nerve palsy and optimize the treatment. In this context, magnetic resonance imaging (MRI) plays an important role in the management of abducens nerve palsy. ${ }^{2}$ In rare cases, pathology involving Dorello's canal, which is the osteofibrous canal in the skull base that includes the abducens nerve and the inferior petrosal sinus (IPS), can cause abducens nerve palsy; however, this is often overlooked. ${ }^{3}$ Here, we present a rare case of a left transverse-sigmoid sinus dural arteriovenous fistula (dAVF) with drainage into the left IPS that presented with isolated left abducens nerve palsy presumably caused by venous hypertension in the left IPS within Dorello's canal.

A previously healthy 64-year-old woman was referred to our hospital due to acute-onset painless diplopia without any associated symptoms, which exacerbated over the course of 1 week. A neurological examination revealed impaired abduction of the left eye with no other abnormal findings (Fig. 1A), which suggested isolated left abducens nerve palsy. Contrastenhanced MRI did not reveal any causative tumor lesions or inflammatory lesions. However, time-of-flight magnetic resonance angiography (TOF-MRA) revealed venous enhancement of the left IPS at the left Dorello's canal, indicating the presence of rapid retrograde venous drainage into the left IPS (Fig. 1B and C). Subsequent cerebral angiography confirmed a left transverse-sigmoid sinus dAVF allowing retrograde venous drainage into the left IPS (Fig. 1D). Embolization of the dAVF alone resolved the enhancement of the left IPS at the left Dorello's canal in TOF-MRA (Fig. 1E) and, subsequently, the left abducens palsy.

Dorello's canal, which was first described by Wenzel Leopold Gruber in 1859 and later by Primo Dorello in 1905, is the osteofibrous canal in the skull base located under Gruber's ligament. ${ }^{4}$ Dorello's canal is bordered by Gruber's ligament superiorly, the dorsum sellae of the sphenoid bone medially, the clivus of the occipital bone inferiorly, and the petrous apex of the temporal bone laterally ${ }^{5}$ (Fig. 1F). Since the abducens nerve runs alongside the IPS within the canal ${ }^{4}$ (Fig. 1G), we should pay attention to Dorello's canal in cases of abducens nerve palsy. A dAVF is an abnormal arteriovenous shunt between the dural arteries and dural venous sinuses or meningeal veins, and presents with various symptoms caused by venous hypertension of the vein involved in the drainage. ${ }^{6}$ Based on the clinical course and radiographical findings in the present case, we believe that venous hypertension due to retrograde venous drainage into the IPS caused by the dAVF damaged the abducens nerve in Dorello's canal. Isolated abducens nerve palsy due to compression of the abducens nerve by the IPS in Dorello's canal is extremely rare, but has been reported in patients with IPS thrombosis ${ }^{6}$ and in those undergoing IPS sampling as part of a workup for Cushing's disease. ${ }^{7}$ Neurologists should pay careful attention to Dorello's canal when evaluating patients with isolated abducens nerve palsy, and investigating this canal using MRI with detailed anatomical knowledge

@ This is an Open Access article distributed under the terms of the Creative Commons Attribution Non-Commercial License (https://creativecommons.org/licenses/by-nc/4.0) which permits unrestricted non-commercial use, distribution, and reproduction in any medium, provided the original work is properly cited. 

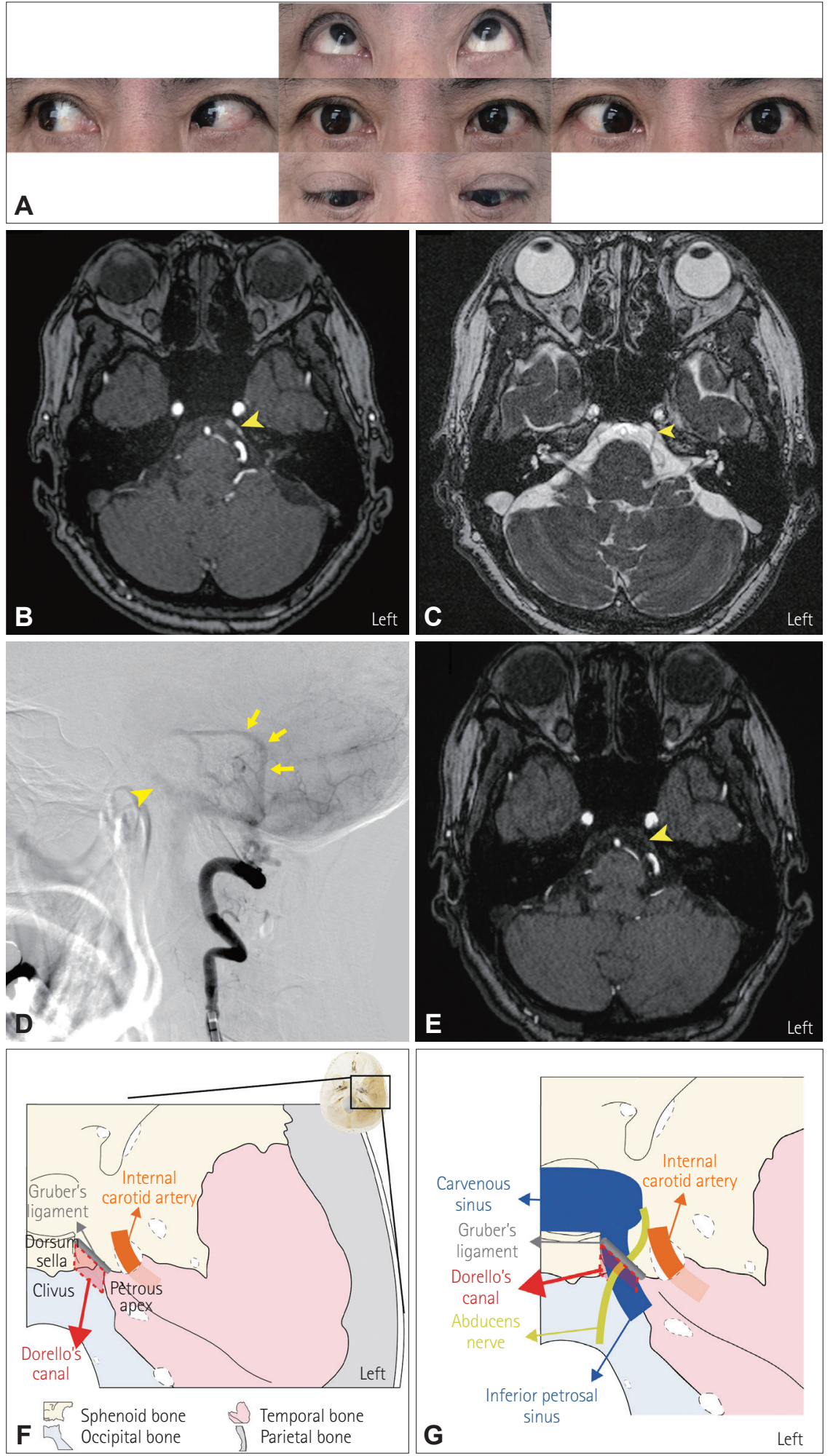

Fig. 1. Imaging findings of the present case and the schematic representation of Dorello's canal. A: The patient presented with impaired abduction of the left eye. B: TOF-MRA image shows venous enhancement of the left IPS in the left Dorello's canal (arrowhead). C: True fast imaging with steady-state precession shows the abducens nerve (arrowhead). D: Angiogram from a left lateral view of the left vertebral artery reveals a dural arteriovenous fistula (arrows) in the left transverse-sigmoid sinus with retrograde drainage into the left IPS (arrowhead). E: Follow-up TOF-MRA image shows resolution of the venous enhancement of the left IPS (arrowhead). F: Schematic representation of the axial view of the skull base shows the anatomical location of Dorello's canal. G: The abducens nerve runs alongside the IPS in Dorello's canal before entering the cavernous sinus. IPS: inferior petrosal sinus, TOF-MRA: time-offlight magnetic resonance angiography. 
of the canal is particularly important.

\section{Conflicts of Interest}

The authors have no potential conflicts of interest to disclose.

\section{Acknowledgements}

We thank Dr. Takahiro Kamada for providing us with the inspiration to write the manuscript. Dr. Kamada died in January 2019, and we wish to dedicate this article to his memory. We also thank Dr. Tomoo Tokime and Dr. Yoshinori Akiyama for helping us to treat the patient.

\section{REFERENCES}

1. Patel SV, Mutyala S, Leske DA, Hodge DO, Holmes JM. Incidence, associations, and evaluation of sixth nerve palsy using a populationbased method. Ophthalmology 2004;111:369-375.

2. Tamhankar MA, Biousse V, Ying GS, Prasad S, Subramanian PS, Lee MS, et al. Isolated third, fourth, and sixth cranial nerve palsies from presumed microvascular versus other causes: a prospective study. Ophthalmology 2013;120:2264-2269.

3. Mittal SO, Siddiqui J, Katirji B. Abducens nerve palsy due to inferior petrosal sinus thrombosis. J Clin Neurosci 2017;40:69-71.

4. Kshettry VR, Lee JH, Ammirati M. The Dorello canal: historical development, controversies in microsurgical anatomy, and clinical implications. Neurosurg Focus 2013;34:E4.

5. Joo W, Yoshioka F, Funaki T, Rhoton AL Jr. Microsurgical anatomy of the abducens nerve. Clin Anat 2012;25:1030-1042.

6. Gandhi D, Chen J, Pearl M, Huang J, Gemmete JJ, Kathuria S. Intracranial dural arteriovenous fistulas: classification, imaging findings, and treatment. AJNR Am J Neuroradiol 2012;33:1007-1013.

7. Lefournier V, Gatta B, Martinie M, Vasdev A, Tabarin A, Bessou P, et al. One transient neurological complication (sixth nerve palsy) in 166 consecutive inferior petrosal sinus samplings for the etiological diagnosis of Cushing's syndrome. J Clin Endocrinol Metab 1999;84:34013402 . 